\title{
Modeling of a Dual Stator Induction Generator with and Without Cross Magnetic Saturation
}

\author{
Marwa Ben Slimene ${ }^{1}$, Mohamed Arbi Khlifi ${ }^{2 *}$, Mouldi Ben Fredj ${ }^{1}$, and Habib Rehaoulia ${ }^{1}$ \\ ${ }^{1}$ University of Tunis, National School of Engineering of Tunis ENSIT \\ ${ }^{2}$ University of Hail, College of Engineering Hail \\ Research Laboratory SIME, ENSIT. Team: ETEC (Conversion and Treatment of Electrical Energy)
}

(Received 3 April 2015, Received in final form 16 June 2015, Accepted 17 June 2015)

\begin{abstract}
This paper discusses general methods of modelling magnetic saturation in steady-state, two-axis (d \& q) frame models of dual stator induction generators (DSIG). In particular, the important role of the magnetic coupling between the $d-q$ axes (cross-magnetizing phenomenon) is demonstrated, with and without cross-saturation. For that purpose, two distinct models of DSIGs, with and without cross-saturation, are specified. These two models are verified by an application that is sensitive to the presence of cross-saturation, to prove the validity of these final methods and the equivalence between all developed models. Advantages of some of the models over the existing ones and their applicability are discussed. In addition, an alternative is given to evaluate all saturation factors (static and dynamic) by just calculating the static magnetizing inductance which is simply the magnitude of the ratio of the magnetizing flux to the current. The comparison between the simulation results of the proposed model with experimental results gives a good correspondence, especially at startup.
\end{abstract}

Keywords : dual stator induction generator, cross magnetic saturation, magnetizing inductance, self-excited DSIG, modeling

\section{Introduction}

Machines with more than three phases have been in use for years in industrial applications, primarily in the high power applications $[1,2]$. The main advantage of increasing the number of phases is that, on one hand, it allows the reduction of the size of the components in power modulators of energy. On the other hand it allows better tolerance and greater reliability [3-6]. Among multiphase machines, the dual stator induction generator whose angular displacement between the two stars is $0^{\circ}, 30^{\circ}$ or $60^{\circ}$ is the most widely used. Such a machine, in addition to the power segmentation and redundancy it carries, has the advantage of reducing the torque pulsations, rotor losses and the reduction of harmonic current [7, 8]. However, the introduction of magnetic nonlinearities in the electrical equations that describe its operation has always been a topical issue for polyphase machines.

\footnotetext{
(C)The Korean Magnetics Society. All rights reserved.

*Corresponding author: Tel: +21626969314

Fax:+21671391166, e-mail: mohamedarbi.khlifi@issatm.rnu.tn/

benslimene.marwa@gmail.com
}

Indeed, taking into account the saturation is not simply dictated by the desire to improve the results, but it can sometimes be a necessity [9].

Besides, developments of vector control methods gave rise to other type of models: the mixed ones. The literature dealing with vector control or field orientation control methods is very rich, but fundamentals are sufficiently summarized in $[10,11]$. Application of such control techniques lead to the use of new models based on stator winding currents and fluxes, stator winding currents and rotor winding fluxes and stator currents associated with magnetizing fluxes. Elsewhere, it has to be emphasized that the $d-q$ modeling theory of induction machines remains the best for performing and analyzing any kind of transient operation, except if local phenomena have to be investigated. For that purpose, as in some cases of diagnosis, the 'abc' frame and the primary equations must be used to handle the known difficulties $[12,13]$.

Many models have been developed to study the behavior of electrical machines. There are models of current or flux and others have been developed for vector control, called mixed models. To study the behavior of the DSIG at startup, we have adopted in this paper a model that 
takes into account the nonlinearity of the magnetic circuit based on the equivalent electric circuit approach $[14,15]$.

In this paper, we propose a dynamic model of a dual stator induction generator (DSIG), with and without magnetic saturation, based on the equivalent electric circuit approach and taking into account the stator mutual leakage inductance between two stars. We develop the model with and without cross saturation and these two models are simulated and compared with the experimental results. Finally the validation of the proposed model has been realized using an experimental test bench setup.

\section{Space Vector Equations}

In the analysis of the transient performance of electrical machines, either of two closely related methods may be used. One of these is the well known matrix method of generalized induction machine theory. In the following, the voltage differential equations for a three-phase machine in terms of the space-vector will be established when saturation of the main flux-path is also considered. The basic model of a dual stator induction generator may be given in terms of space vectors, in an arbitrary frame of reference $\omega_{a}$, with the following set of equations:

$$
\begin{aligned}
& V_{d s 1}=R_{s} i_{d s 1}+\frac{d \lambda_{d s 1}}{d t}-w_{a} \lambda_{q s 1} \\
& V_{q s 1}=R_{s} i_{q s 1}+\frac{d \lambda_{q s 1}}{d t}+w_{a} \lambda_{d s 1} \\
& V_{d s 2}=R_{s} i_{d s 2}+\frac{d \lambda_{d s 2}}{d t}-w_{a} \lambda_{q s 2} \\
& V_{q s 2}=R_{s} i_{q s 2}+\frac{d \lambda_{q s 2}}{d t}+w_{a} \lambda_{d s 2} \\
& 0=R_{r} i_{d r}+\frac{d \lambda_{d r}}{d t}-\left(w_{a}-w\right) \lambda_{q r} \\
& 0=R_{r} i_{q r}+\frac{d \lambda_{q r}}{d t}+\left(w_{a}-w\right) \lambda_{d r}
\end{aligned}
$$

where $w_{a}$ is the speed of the reference frame. The expressions of stator and rotor flux linkages are:

$$
\left\{\begin{array}{l}
\lambda_{d s 1}=\left(l_{s}+l_{s m}\right) i_{d s 1}+l_{s m} i_{d s 2}+\lambda_{d m} \\
\lambda_{q s 1}=\left(l_{s}+l_{s m}\right) i_{q s 1}+l_{s m} i_{q s 2}+\lambda_{q m} \\
\lambda_{d s 2}=l_{s m} i_{d s 1}+\left(l_{s}+l_{s m}\right) i_{d s 2}+\lambda_{d m} \\
\lambda_{q s 2}=l_{s m} i_{q s 1}+\left(l_{s}+l_{s m}\right) i_{q s 2}+\lambda_{q m} \\
\lambda_{d r}=l_{r} i_{d r}+\lambda_{d m} \\
\lambda_{q r}=l_{r} i_{q r}+\lambda_{q m}
\end{array}\right.
$$

Where:

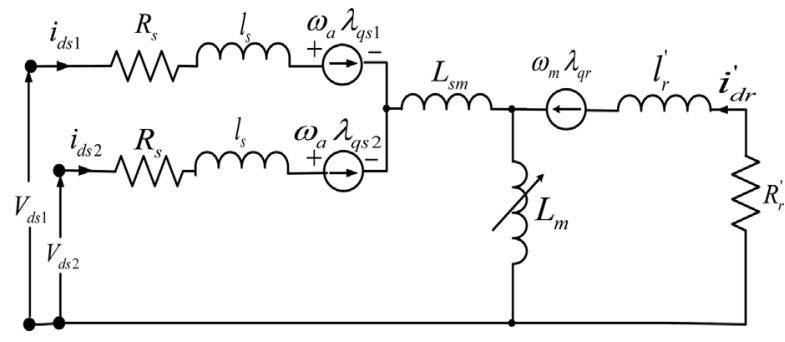

Fig. 1. Equivalent circuit of DSIG.

$$
\left\{\begin{array}{l}
i_{d m}=i_{d s 1}+i_{d s 2}+i_{d r} \\
i_{q m}=i_{q s 1}+i_{q s 2}+i_{q r}
\end{array}\right.
$$

where $l_{s m}$ is the common mutual leakage inductance between the two sets of stators windings, $l_{s}, l_{r}$ are the stator and rotor leakage inductance respectively, and $L_{m}$ is the mutual inductance between the stator and rotor.

The electromagnetic torque and dynamic equations can be expressed as:

$$
\begin{aligned}
& C_{e m}=n_{p} \frac{L_{m}}{L_{r}}\left[\left(i_{q s 1}+i_{q s 2}\right) \lambda_{d r}-\left(i_{d s 1}+i_{d s 2}\right) \lambda_{q r}\right] \\
& J \frac{d w}{d t}+K_{f} w=n_{p}\left(C_{e m}-C_{r}\right)
\end{aligned}
$$

In Fig. 1, with saturation neglected, we have:

$$
\left\{\begin{array}{l}
\lambda_{d m}=L_{m} i_{d m} \\
\lambda_{q m}=L_{m} i_{q m}
\end{array}\right.
$$

To the contrary, the alternative that we propose in this contribution begins with the fitting of the characteristic relating the magnetizing inductance $L_{m}$, as obtained experimentally, to the air gap flux. Fig. 2 shows such a
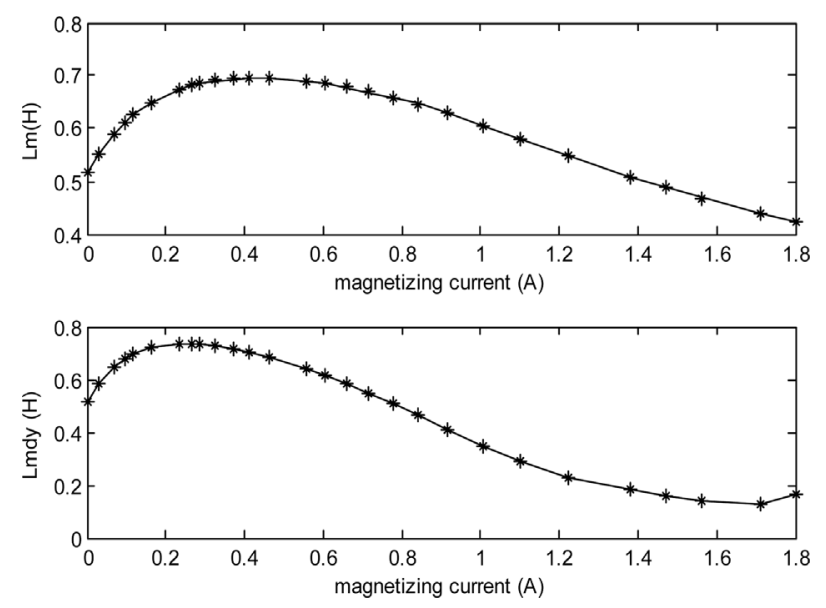

Fig. 2. Approximation of magnetizing curve and measured static and dynamic magnetizing inductances. 
characteristic which, in general, possesses two portions. One corresponds to the unsaturated zone where $L_{m}$ is constant and all the dynamic terms are null; the other corresponds to the saturated region and may be represented by a limited number of segments. So we have:

$$
\left\{\begin{array}{l}
\lambda_{m}=\sqrt{\lambda_{m d}^{2}+\lambda_{m q}^{2}} \\
i_{m}=\sqrt{i_{m d}^{2}+i_{m q}^{2}}
\end{array}\right.
$$

\section{Method Description and Derivation of Saturated Models}

\subsection{The Approach to the Main Flux Saturation Mod-} eling in DSIG

This becomes a necessary consideration for the study of an autonomous dual stator induction generator because the linear model is not able to describe the behavior of the system. Thus, only approaches that take account the saturation effect can be utilized. This effect is not easy to simulate using two-three phase classical models. So, we usually adopt two-diphase approaches to globally take account of the magnetic non-linearity.

Thus, the saturation effect is taken into account through the expression of the static and dynamic magnetizing inductances with respect to the magnetizing current. They are evaluated from the open circuit d-axis magnetizing curve $\lambda_{m}=f\left(i_{m}\right)$.

$$
L_{m}=\frac{\lambda_{m}}{i_{m}} \quad L_{m d y}=\frac{d \lambda_{m}}{d i_{m}}
$$

The static magnetizing inductance versus the magnetizing current that is shown in Fig. 3 is approximated by a polynomial of degree 7 as follows:

$$
\begin{aligned}
& L_{m}=k_{1} i_{m}^{7}+k_{2} i_{m}^{6}+k_{3} i_{m}^{5}+k_{4} i_{m}^{4}+k_{5} i_{m}^{3}+k_{6} i_{m}^{2}+k_{7} i_{m}+k_{8} \\
& \text { with } \\
& k_{1}=0.19303 ; \quad k_{2}=-1.4276 ; \\
& k_{3}=4.3069 ; \quad k_{4}=-6.8637 \\
& k_{5}=6.4026 ; \quad k_{6}=-3.8101 ; \\
& k_{7}=1.2896 ; \quad k_{8}=0.51665 ;
\end{aligned}
$$

The leakage inductances in equations (7) are assumed to be constant and only the $\mathrm{d}-\mathrm{q}$ magnetizing flux is subject to saturation. Deriving stator and rotor linkage fluxes, in equations (7), leads to constancy with respect to time, only the main flux derivative of the $d$-q magnetizing flux $\lambda_{d m}, \lambda_{q m}$.

$$
\left\{\begin{array}{l}
\cos \beta=\frac{\lambda_{d m}}{\lambda_{m}}=\frac{i_{d m}}{i_{m}} \\
\sin \beta=\frac{\lambda_{q m}}{\lambda_{m}}=\frac{i_{q m}}{i_{m}}
\end{array}\right.
$$

Angular $\beta$ designates the position of $\lambda_{m}$ with respect to the d-q axis. It also characterizes the position of the magnetizing current $i_{m}$ in the air gap.

Therefore, $d \lambda_{d m} / d t, d \lambda_{q m} / d t$ have to be described by means of the winding current.

$$
\left\{\begin{array}{l}
\frac{d \lambda_{d m}}{d t}=\frac{d\left(\lambda_{m} \cos \beta\right)}{d t}=\cos \beta \frac{d \lambda_{m}}{d t}-\lambda_{m} \sin \beta \frac{d \beta}{d t} \\
\frac{d \lambda_{q m}}{d t}=\frac{d\left(\lambda_{m} \sin \beta\right)}{d t}=\sin \beta \frac{d \lambda_{m}}{d t}+\lambda_{m} \cos \beta \frac{d \beta}{d t}
\end{array}\right.
$$
Terms, $\cos \beta \frac{d \lambda_{m}}{d t}$ and $\sin \beta \frac{d \lambda_{m}}{d t}$ may be elaborated as
follows:

$$
\left\{\begin{array}{l}
\cos \beta \frac{d \lambda_{m}}{d t}=L_{m d y} \frac{d i_{d m}}{d t}+L_{m d y} i_{q m} \frac{d \beta}{d t} \\
\sin \beta \frac{d \lambda_{m}}{d t}=L_{m d y}\left(\frac{d i_{q m}}{d t}-i_{q m} \frac{\cos \beta}{\sin \beta} \frac{d \alpha}{d t}\right)
\end{array}\right.
$$

Therefore (16) is written:

$$
\begin{aligned}
& \left\{\begin{array}{l}
\frac{d \lambda_{d m}}{d t}=L_{m d y} \frac{d i_{d m}}{d t}+\left(i_{q m} L_{m d y}-\sin \beta \lambda_{m}\right) \frac{d \beta}{d t} \\
\frac{d \lambda_{q m}}{d t}=L_{m d y} \frac{d i_{q m}}{d t}+\left(\lambda_{m} \cos \beta-i_{q m} \frac{\cos \beta}{\sin \beta}\right) \frac{d \beta}{d t}
\end{array}\right. \\
& \beta=\tan ^{-1}\left(\frac{i_{q m}}{i_{d m}}\right)
\end{aligned}
$$

Thus

$$
\frac{d \beta}{d t}=\left(\frac{\cos \beta}{i_{m}} \frac{d i_{q m}}{d t}-\frac{\sin \beta}{i_{m}} \frac{d i_{d m}}{d t}\right)
$$

We can write:

$$
\begin{aligned}
& \left\{\begin{array}{l}
\frac{d \lambda_{d m}}{d t}=L_{d} \frac{d i_{d m}}{d t}+L_{d q} \frac{d i_{q m}}{d t} \\
\frac{d \lambda_{q m}}{d t}=L_{d q} \frac{d i_{d m}}{d t}+L_{q} \frac{d i_{q m}}{d t}
\end{array}\right. \\
& \left\{\begin{array}{l}
L_{d}=L_{m}+\cos ^{2} \beta\left(L_{m d y}-L_{m}\right) \\
L_{q}=L_{m}+\sin ^{2} \beta\left(L_{m d y}-L_{m}\right) \\
L_{d q}=\cos \beta \sin \beta\left(L_{m d y}-L_{m}\right)
\end{array}\right.
\end{aligned}
$$




\subsection{Derivation of the winding current models}

Based on the general equation of the self-excited dual stator induction generator (SEIG), a mathematical model is developed to represent the dynamic characteristic involved in the voltage build up of the dual stator SEIG. The dynamic analysis has to demonstrate the transient voltage and frequency developed by the induction generator.

After deriving the system (7) and by introducing equation (21) in (7), we can obtain the following system:

$$
\left\{\begin{array}{l}
\frac{d \lambda_{d s 1}}{d t}=\left(l_{s}+l_{s m}\right) \frac{d i_{d s 1}}{d t}+l_{s m} \frac{d i_{d s 2}}{d t}+L_{d} \frac{d i_{d m}}{d t}+L_{d q} \frac{d i_{q m}}{d t} \\
\frac{d \lambda_{q s 1}}{d t}=\left(l_{s}+l_{s m}\right) \frac{d i_{q s 1}}{d t}+l_{s m} \frac{d i_{q s 2}}{d t}+L_{q} \frac{d i_{q m}}{d t}+L_{d q} \frac{d i_{d m}}{d t} \\
\frac{d \lambda_{d s 2}}{d t}=l_{s m} \frac{d i_{d s 1}}{d t}+\left(l_{s}+l_{s m}\right) \frac{d i_{d s 2}}{d t}+L_{d} \frac{d i_{d m}}{d t}+L_{d q} \frac{d i_{q m}}{d t} \\
\frac{d \lambda_{q s 2}}{d t}=l_{s m} \frac{d i_{q s 1}}{d t}+\left(l_{s}+l_{s m}\right) \frac{d i_{q s 2}}{d t}+L_{q} \frac{d i_{q m}}{d t}+L_{d q} \frac{d i_{d m}}{d t} \\
\frac{d \lambda_{d r}}{d t}=l_{r} \frac{d i_{q r}}{d t}+L_{d} \frac{d i_{d m}}{d t}+L_{d q} \frac{d i_{q m}}{d t} \\
\frac{d \lambda_{q r}}{d t}=l_{r} \frac{d i_{q r}}{d t}+L_{q} \frac{d i_{q m}}{d t}+L_{d q} \frac{d i_{d m}}{d t}
\end{array}\right.
$$

The main differential system can be represented by

$$
[V]=[A][\dot{X}]+[B][X]
$$

With:

$$
\begin{gathered}
{[V]=\left[\begin{array}{llllll}
V_{d s 1} & V_{q s 1} & V_{d s 2} & V_{q s 2} & 0 & 0
\end{array}\right]^{t}} \\
{[X]=\left[\begin{array}{lllllll}
i_{d s 1} & i_{q s 1} & i_{d s 2} & i_{q s 2} & i_{d r} & i_{q r}
\end{array}\right]^{t}} \\
{[A]=\left[\begin{array}{cccccc}
l_{s}+l_{s m}+L_{d} & L_{d q} & l_{s m}+L_{d} & L_{d q} & L_{d} & L_{d q} \\
L_{d q} & l_{s}+l_{s m}+L_{q} & L_{d q} & l_{s m}+L_{q} & L_{d q} & L_{q} \\
l_{s m}+L_{d} & L_{d q} & l_{s}+l_{s m}+L_{d} & L_{d q} & L_{d} & L_{d q} \\
L_{d q} & l_{s m}+L_{q} & L_{d q} & l_{s}+l_{s m}+L_{q} & L_{d q} & L_{q} \\
L_{d} & L_{d q} & L_{d} & L_{d q} & l_{r}+L_{d} & L_{d q} \\
L_{d q} & L_{q} & L_{d q} & L_{q} & L_{d q} & l_{r}+L_{q}
\end{array}\right]} \\
{[B]=\left[\begin{array}{ccccccc}
R_{s} & 0 & 0 & 0 & 0 & 0 \\
0 & R_{s} & 0 & 0 & 0 & 0 \\
0 & 0 & R_{s} & 0 & 0 & 0 \\
0 & 0 & 0 & R_{s} & 0 & 0 \\
0 & w L_{q m} & 0 & w L_{q m} & R_{r} & w\left(l_{r}+L_{q m}\right) \\
-w L_{d m} & 0 & -w L_{d m} & 0 & w\left(l_{r}+L_{d m}\right) & R_{r}
\end{array}\right]}
\end{gathered}
$$

\section{Saturated Model without Cross-Saturation}

If magnetic saturation is accounted for, the relation

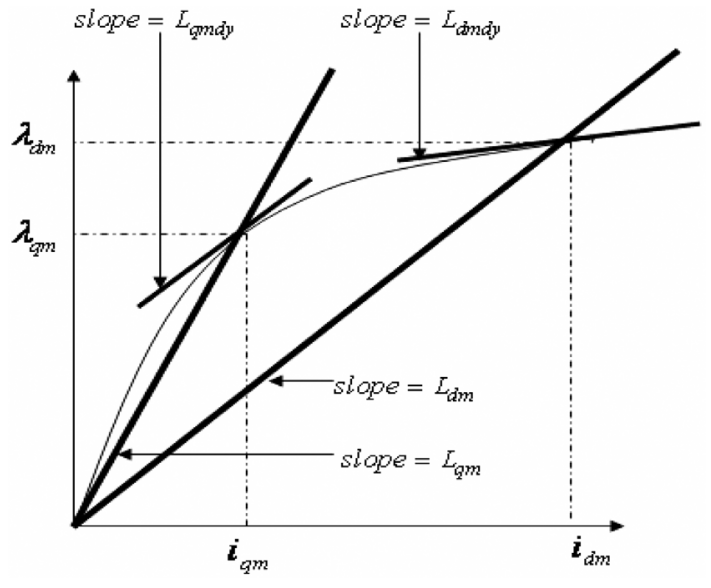

Fig. 3. Static and dynamic mutual inductances for both $d$ and q axes.

binding the magnetizing current and the magnetizing flux is nonlinear, as Fig. 2 indicates. To avoid cross-saturation complexities, in the main equations the magnetic saturation is introduced independently along the $\mathrm{d}$ and $\mathrm{q}$ axes. In addition, since the air-gap is uniform the no load magnetization curve is valid for both axes. With this latter circumstance one can define the static and dynamic mutual inductances as in Fig. 3.

$$
\begin{aligned}
& L_{d m}=\frac{\lambda_{d m}}{i_{d m}}, L_{q m}=\frac{\lambda_{q m}}{i_{q m}} \\
& L_{d m d y}=\frac{d \lambda_{d m}}{d i_{d m}}, L_{q m d y}=\frac{d \lambda_{q m}}{d i_{q m}}
\end{aligned}
$$

The derivation of the $\left(\bar{i}_{s 1}, \bar{i}_{s 2}, \bar{i}_{r}\right)$ model requires the description of fluxes $\bar{\lambda}_{s 1}, \bar{\lambda}_{s 2}$ and $\bar{\lambda}_{r}$ as well as their derivatives in terms of the chosen state variables. Deriving stator and rotor linkage fluxes, we get:

$$
\left\{\begin{array}{l}
\frac{d \lambda_{d s 1}}{d t}=\left(l_{s}+l_{s m}\right) \frac{d i_{d s 1}}{d t}+l_{s m} \frac{d i_{d s 2}}{d t}+L_{d m d y} \frac{d i_{d m}}{d t} \\
\frac{d \lambda_{q s 1}}{d t}=\left(l_{s}+l_{s m}\right) \frac{d i_{q s 1}}{d t}+l_{s m} \frac{d i_{q s 2}}{d t}+L_{q m d y} \frac{d i_{q m}}{d t} \\
\frac{d \lambda_{d s 2}}{d t}=l_{s m} \frac{d i_{d s 1}}{d t}+\left(l_{s}+l_{s m}\right) \frac{d i_{d s 2}}{d t}+L_{d m d y} \frac{d i_{d m}}{d t} \\
\frac{d \lambda_{q s 2}}{d t}=l_{s m} \frac{d i_{q s 1}}{d t}+\left(l_{s}+l_{s m}\right) \frac{d i_{q s 2}}{d t}+L_{q m d y} \frac{d i_{q m}}{d t} \\
\frac{d \lambda_{d r}}{d t}=l_{r} \frac{d i_{q r}}{d t}+L_{d m d y} \frac{d i_{d m}}{d t} \\
\frac{d \lambda_{q r}}{d t}=l_{r} \frac{d i_{q r}}{d t}+L_{q m d y} \frac{d i_{q m}}{d t}
\end{array}\right.
$$

Finally the voltage equations of the $\left(\bar{i}_{s 1}, \bar{i}_{s 2}, \bar{i}_{r}\right)$ model are: 


$$
\begin{aligned}
& {[V]=\left[\begin{array}{llllll}
V_{d s 1} & V_{q s 1} & V_{d s 2} & V_{q s 2} & 0 & 0
\end{array}\right]^{t}} \\
& {[X]=\left[\begin{array}{llllll}
i_{d s 1} & i_{q s 1} & i_{d s 2} & i_{q s 2} & i_{d r} & i_{q r}
\end{array}\right]^{t}} \\
& {[A]=\left[\begin{array}{ccc}
l_{s}+l_{s m}+L_{d m d y} & 0 & l_{s m}+L_{d m d y} \\
0 & l_{s}+l_{s m}+L_{q m d y} & 0 \\
l_{s m}+L_{d m d y} & 0 & l_{s}+l_{s m}+L_{d m d y} \\
0 & l_{s m}+L_{q m d y} & 0 \\
L_{d m d y} & 0 & L_{d m d y} \\
0 & L_{q m d y} & 0
\end{array}\right.} \\
& \begin{array}{ccc}
0 & L_{d m d y} & 0 \\
l_{s m}+L_{q m d y} & 0 & L_{q m d y} \\
0 & L_{d m d y} & 0 \\
l_{s}+l_{s m}+L_{q m d y} & 0 & L_{q m d y} \\
0 & l_{r}+L_{d m d y} & 0 \\
L_{q m d y} & 0 & l_{r}+L_{q m d y}
\end{array} \\
& {[B]=\left[\begin{array}{cccccc}
R_{s} & 0 & 0 & 0 & 0 & 0 \\
0 & R_{s} & 0 & 0 & 0 & 0 \\
0 & 0 & R_{s} & 0 & 0 & 0 \\
0 & 0 & 0 & R_{s} & 0 & 0 \\
0 & w L_{q m} & 0 & w L_{q m} & R_{r} & w\left(l_{r}+L_{q m}\right) \\
-w L_{d m} & 0 & -w L_{d m} & 0 & w\left(l_{r}+L_{d m}\right) & R_{r}
\end{array}\right]}
\end{aligned}
$$

\section{Experimental Results}

Though the theory of main flux saturation is well recognized, an application sensitive to the presence of saturation on DSIG was added to verify the validity of the proposed saturated models and the equivalence between them. The stator of each machine is rewound specifically for the task with two sets of three phase windings (two stars), spatial shifting $\theta=0^{\circ}$ between the two stars. For

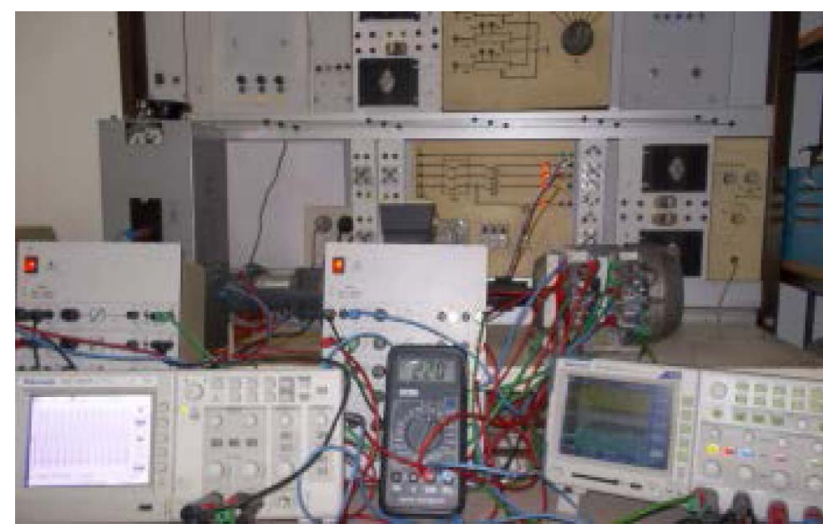

Fig. 4. (Color online) Photo of experimental set-up. that purpose a classical application is selected. The application treats the self excitation of an isolated DSIG. The machine is star connected for all tests. The saturation curve was measured with the machine driven at synchronous speed. A photo of experimental set-up representing the applications tests is provided as Fig. 4.

Figs. 5 and 6 respectively simulate at load the generated voltage and stator current per phase, and the winding voltage and winding current per phase at the rated speed and with $\mathrm{C}=12 \mu \mathrm{F}$. Results are obtained using any of the possible models, in particular with those fully developed in this paper: current model $\left(\bar{i}_{s 1}, \bar{i}_{s 2}, \bar{i}_{r}\right)$. This result proves the validity of the unified method and the equivalence of both models with and without cross saturation. It comes from the main sets of equations Eqs. (23) and (31), that the first model containing cross-coupling terms $\mathrm{L}_{\mathrm{dq}}$ is the heaviest to compute. All its matrix [A] elements are nonnull and depend on magnetic saturation, Eq. (27). Moreover, the simulation time is mainly related to the shape of the system matrix $[\mathrm{A}]$. The more zeros it contains the shorter the simulation time. In the model without crossmagnetizing the magnetic saturation is incorporated independently along the $\mathrm{d}$ and $\mathrm{q}$ axes in the set of dynamic (a)

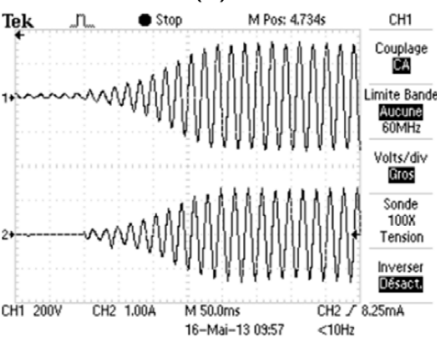

(b)

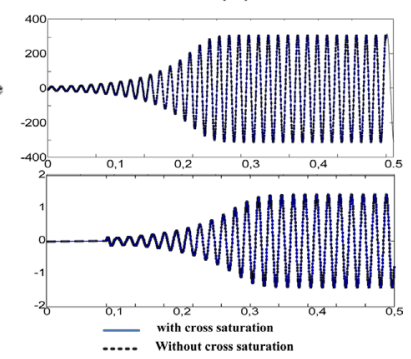

Fig. 5. (Color online) Generated voltage and stator current per phase 'a' set 'II', for an excited dual induction generator with load, rated speed and Capacitor $(\mathrm{C}=12 \mu \mathrm{F})$ per phase. (a) experimental (b) simulation (with and without cross saturation). (a)

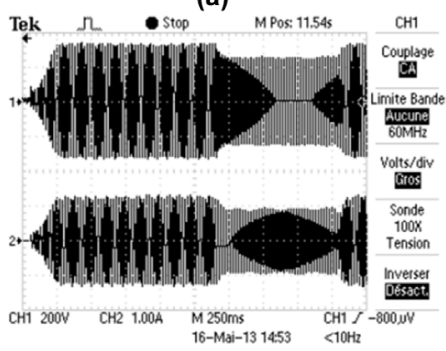

(b)

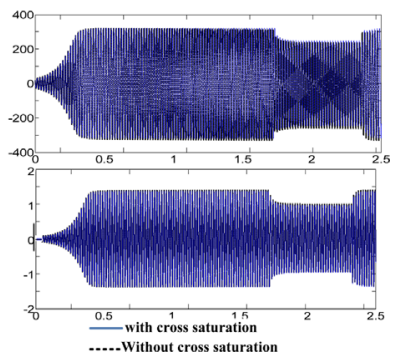

Fig. 6. (Color online) Build-up of winding voltage, winding current per phase 'a' set 'I', for an excited dual induction generator with load, rated speed and Capacitor $(\mathrm{C}=12 \mu \mathrm{F})$ per phase. (a) experimental (b) simulation (with and without cross 
equations. This makes our system model representing by Eq. (34) simpler and less time consuming.

The results of the dynamic self-excitation process given in the previous figures are based on remnant or residual flux in the iron core providing the initial condition required by the self-excited DSIG. When the initial conditions for self excitation are satisfied the flux grows and associated with the growth of flux linkage the generated voltage also grows. As discussed previously the selfexcitation process can also be initiated with a charged capacitor. A charged capacitor will provide magnetizing current to the dual stator induction generator and the flux and the terminal voltage will grow. When the self-excitation process is started from a charged capacitor there is a step voltage at the moment the capacitors are connected to the terminals of the DSIG and that provides a transient exciting current.

In the model without cross-saturation the magnetic saturation is incorporated independently along the $\mathrm{d}$ and $\mathrm{q}$ axes in the set of dynamic equations. This makes our system model represented by Eq. (34), be simpler and less time consuming when it has to implemented in a DSP. This fact is confirmed by the simulation time between two models, where we have computed the time of execution of the two. Half of its elements are zeros, enabling faster simulation. Consequently, the new model, without $\mathrm{d}-\mathrm{q}$ cross-saturation, is more advantageous and its use is thus advised.

\section{Conclusion}

In this paper, the modeling of dual stator induction generators is presented, with and without cross saturation. The impact of the mutual coupling Ldq due to the interaction of orthogonal axes is represented by the computational inductances $\mathrm{Ld}$ and $\mathrm{Lq}$ which depend on both saturation and on the reference frame. The decomposition onto orthogonal axes is necessary for computational efficiency and not for physical reasons.

With the considered $(\mathrm{d}, \mathrm{q})$ frame the equations assume specific forms for the analysis of a given steady-state or transient regime. The characteristics contained in the paper show in a convincing manner that, for high saturation, application of the method with cross saturation is compulsory. Even the simplified method without cross saturation ( $\mathrm{Ldq}$ is ignored) is preferable. It can also be seen that for normal saturation the results obtained by all these method are quite close and can be accepted.

\section{References}

[1] M. Ben Slimene, M. A. Khlifi, M. Benfredj, and H. Rehaoulia, Journal of Circuits, Systems, and Computers 24, 1450169 (2015).

[2] M. Archana, S. Nanoty, and A. R. Chudasama, IEEE Electric Ship Technologies Symposium (ESTS) 42, 283 (2011).

[3] T. A. Lipo, International Conference on Electrical Machines, Athens, Greece 860 (1980).

[4] R. Bojoi, M. Lazzari, F. Profumo, and A. Tenconi, IEEE Transactions on Industry Applications 39, 752 (2003).

[5] E. Levi, IEEE Trans. Indus. Electronics 55, 1893 (2008).

[6] A. Tessarolo, 5th IET Int. Conf. on Power Electronics, Machines and Drives (PEMD). 1 (2010).

[7] O. Olorunfemi and W. Zhiqiao, IEEE Trans. Indus. Applications 44, 1099 (2008).

[8] R. N. Andriamalala, H. Razik, and G. Didier, IEEE Industrial Application Society Annual Meeting-IAS, Tampa, Florida, USA (2006).

[9] M. A. Khlifi and H. Rehaoulia, Int. J. Phys. Sci. 6, 4928 (2011).

[10] E. Levi, IEEE Trans. Energy Conver 12, 211 (1997).

[11] K. Idjdarene, D. Rekiou, T. Rekioua, and A. Tounzi, Energy Conversion and Management 49, 2609 (2008).

[12] S. Nandi, IEEE Trans. on Ind. Applic. 40, 1302 (2004).

[13] D. Joshi and K. S. Sandhu, International Journal of Mathematical Models and Methods in Applied Sciences 3, 68 (2009).

[14] H. Amimeur, D. Aouzellag, R. Abdessemed, and K. Ghedamsi, Electrical Power and Energy Systems 42, 60 (2012).

[15] G. K. Singh, A. Senthil, and R. P. Saini, European Transactions on Electrical Power 20, 884 (2010). 